\title{
INTERSECTION REPRESENTATION OF DIGRAPHS \\ IN TREES WITH FEW LEAVES
}

\author{
In-Jen Lin $^{1}$ \\ National Ocean University, Taipei, Taiwan, ijlin@tiger.cs.nthu.edu.tw \\ Malay K. Sen ${ }^{2}$ \\ North Bengal University, Darjeeling, India \\ Douglas B. West ${ }^{3}$ \\ University of Illinois, Urbana, IL 61801-2975, west@math.uiuc.edu
}

\begin{abstract}
The leafage of a digraph is the minimum number of leaves in a host tree in which it has a subtree intersection representation. We discuss bounds on the leafage in terms of other parameters (including Ferrers dimension), obtaining a string of sharp inequalities.
\end{abstract}

\section{INTRODUCTION}

An intersection representation of a digraph $D$ assigns an ordered pair $\left(S_{v}, T_{v}\right)$ to each vertex $v \in V(D)$ such that $u v \in E(D)$ if and only if $S_{u} \cap T_{v} \neq \varnothing$. We call $S_{v}$ and $T_{v}$ the source set and sink set of $v$. This model was first described by Beineke and Zamfirescu [1] under the name connection digraph. An essentially equivalent model in terms of bipartite graphs was introduced by Harary, Kabell, and McMorris [7].

When each set in an intersection representation is a subtree of a fixed host tree, we have a subtree representation. Every $n$-vertex digraph has a subtree representation in a star with $n$ leaves. Not every digraph has a subtree representation in a path; those that do are the interval digraphs, which are characterized in $[15,16]$. We define the leafage $l(D)$ of a digraph $D$ to be the minimum number of leaves in a host tree in which $D$ has a subtree representation. Thus leafage is a measure of distance from an interval digraph, and the subtree representations in stars show that $l(D) \leq n(D)$. An analogous parameter

${ }^{1}$ Supported by a University of Illinois Research Board Grant.

2 Supported by the Council for International Exchange of Scholars.

3 Supported by NSA/MSP Grant MDA904-93-H-3040.

Running head: REPRESENTATION OF DIGRAPHS

AMS codes: 05C35, 05C50, 05C75

Keywords: leafage, digraph, intersection representation, Ferrers dimension

Written September 1992, revised July 1997. 
for chordal (undirected) graphs is studied in [8]. Further results about adjacency matrices of interval digraphs appear in $[9,10,15,16,17]$.

We obtain lower bounds on leafage using the idea of Ferrers dimension. The successors of a vertex $v$ are $\{u \in V(D): v u \in E(D)\}$; the predecessors are $\{u \in V(D): u v \in E(D)\}$. A digraph is a Ferrers digraph [14] if its successor sets are linearly ordered by inclusion, which is equivalent to the adjacency matrix $A(D)$ having no 2 by 2 permutation submatrix. Viewing a digraph as a relation $D \subseteq V(D) \times V(D)$, the Ferrers dimension $f(D)$ of $D$ is the minimum number of Ferrers digraphs on $V(D)$ whose intersection is $D$ (introduced in [2]). Since the complement in $V \times V$ of a Ferrers digraph is also a Ferrers digraph, this also equals the minimum number of Ferrers digraphs whose union is $\bar{D}$.

Interval digraphs all have Ferrers dimension at most 2; a digraph $D$ is an interval digraph if and only if $\bar{D}$ is the union of two disjoint Ferrers digraphs [15]. This generalizes to a lower bound on $l(D)$ using Ferrers digraphs. Let $f^{*}(D)$ denote the minimum number of pairwise disjoint Ferrers digraphs whose union is $\bar{D}$. These are Ferrers digraphs whose intersection is $D$ and whose pairwise unions are $V(D) \times V(D)$. Having imposed an extra condition on the minimization, we have $f^{*}(D) \geq f(D)$; we prove that $l(D) \geq f^{*}(D)$.

On the upper side, we study the related catch leafage $l^{*}(D)$ of a digraph $D$. This is the minimum number of leaves in a host tree in which $D$ has a subtree representation such that each sink subtree is a single vertex. (Such representations, particularly when the host tree is a path, are studied in $[12,13,15]$.) This condition restricts the allowable representations, so $l^{*}(D) \geq l(D)$. We prove that $l^{*}(D) \leq w(P(D))$, where $w(P(D))$ is the width of the inclusion poset $P(D)$ on the sets whose incidence vectors are the columns of the adjacency matrix $A(D)$. We also give a sufficient condition for equality in this bound.

We thus obtain the chain of inequalities

$$
f(D) \leq f^{*}(D) \leq l(D) \leq l^{*}(D) \leq w(P(D)) \leq n(D)
$$

We present examples to show that each inequality is best possible. We also present examples to show that each bound is arbitrarily weak, as any one of these parameters can be at most 3 when the next parameter is arbitrarily large.

The upper bound $w(P(D))$ is easily computable, but the lower bounds are not. Cogis [2] and Doignon, Ducamp, and Falmagne [4] proved an easily testable characterization of the digraphs with Ferrers dimension at most 2, but Yannakakis [18] proved that recognition of Ferrers dimension 3 is NP-complete. Müller [11] found a polynomial-time recognition algorithm for interval digraphs (leafage 2). Other than this, we do not know the complexity of recognizing digraphs with bounded values for any of $\left\{f^{*}(D), l(D), l^{*}(D)\right\}$.

\section{SUBTREE REPRESENTATIONS AND LEAFAGE}

We use $u \rightarrow v$ to denote the successor relation; $u \rightarrow v$ means " $u v$ is an edge". A branch point of a tree is a vertex of degree at least 3. We show first that leafage is well-defined.

THEOREM 1. If $D$ is a digraph with $n$ vertices, then $D$ has a subtree representation in a star with at most $n$ leaves. 
Proof: In a star $H$ with $n$ leaves, assign distinct leaves as sink sets for the $n$ vertices. For each $v \in V(D)$, let $S_{v}$ be the star induced by the center of $H$ and the leaves corresponding to the successors of $v$. Then $u \rightarrow v$ if and only if $T_{v} \subseteq S_{u}$, and hence this is a representation.

The bound $l(D) \leq n(D)$ is sharp, as it holds with equality for the digraph $D_{n}$ in Theorem 2. Our tool for proving lower bounds on $l(D)$ is a property of subtrees of a tree. If $T_{i}, T_{j}, T_{k}$ are subtrees of a tree, then we say that $T_{k}$ is between $T_{i}$ and $T_{j}$ if $T_{i} \cap T_{j}=\varnothing$ and the unique path from $T_{i}$ to $T_{j}$ contains a vertex of $T_{k}$ (possibly at the start or end). A collection of pairwise disjoint subtrees having the property that none is between two others is an asteroidal collection of subtrees.

LEMMA 1. If $T_{1}, \ldots, T_{n}$ is an asteroidal collection of subtrees of a tree $T$, then $T$ has at least $n$ leaves.

Proof: We may assume that the path from any leaf of $T$ to the nearest branch point contains a vertex of some $T_{i}$; otherwise, we could delete the vertices before the branch point to reduce the number of leaves without changing the hypotheses. For each leaf $v$ of $T$, we assign to $v$ the first subtree encountered on the path from $v$ to its nearest branch point. If $T$ has fewer than $n$ leaves, then some subtree $T_{k}$ in our list is not assigned to any leaf. Let $x$ be a vertex of $T_{k}$, and let $P$ be a maximal path containing $x$. The endpoints of $P$ are leaves of the tree, and $T_{k}$ is between the subtrees assigned to those leaves. Hence $T$ must have at least $n$ leaves.

LEMMA 2. If $v, w$ have a common successor $u$ that is not a successor of $z$ in a digraph $D$, then $S_{z}$ is not between $S_{v}$ and $S_{w}$ in any subtree representation of $D$. Similarly, if $v, w$ have a common predecessor $u$ that is not a predecessor of $z$ in $D$, then $T_{z}$ is not between $T_{v}$ and $T_{w}$ in any subtree representation of $D$.

Proof: If $S_{z}$ is between $S_{v}$ and $S_{w}$, then $S_{v} \cap S_{w}=\varnothing$, and $T_{u}$ must contain the unique path from $S_{v}$ to $S_{w}$ in the host. This contradicts $S_{z} \cap T_{u}=\varnothing$, since $S_{z}$ has a vertex on this path. The proof of the other statement is similar.

Subtrees of a tree satisfy the Helly property; the members of a pairwise intersecting family of (sub)trees have a common vertex (see, for example, [6, p. 92]).

LEMMA 3. If in a subtree representation of $D$ the source subtrees are pairwise intersecting and the sink subtrees are pairwise intersecting, then $A(D)$ has a row of 1 's or a column of 0's, and similarly $A(D)$ has a column of 1's or a row of 0's.

Proof: In such a representation, the source subtrees have a common vertex, and the sink subtrees have a common vertex. Let $s, t$ denote these vertices, respectively. If $s=t$, then $A(D)$ is all 1's and the claim holds. If $s \neq t$, let $x$ be the vertex of $\bigcup S_{i}$ that is closest to $t$ on the unique $s, t$-path in $T$. Suppose $x \in S_{k}$. If $A(D)$ has no row of 1 's, then $x \neq t$ and some sink subtree $T_{j}$ fails to contain $x$. However, $t \in T_{j}$, and hence $T_{j}$ intersects no source subtree, forcing a column of 0 's in $A(D)$. The other claim follows by considering the vertex of $\bigcup T_{j}$ that is closest to $s$ on the $s, t$-path in $T$. 
Because permuting rows or columns is simply a relabeling of source or sink sets, leafage can be viewed as a property of a 0,1-matrix (the adjacency matrix $A(D)$ ) rather than a property of a digraph. We next show that asteroidal collections are forced by complements of permutation matrices.

THEOREM 2. For $n \geq 3$, let $D_{n}$ be the digraph such that $A\left(D_{n}\right)=J-I$, where $J$ is the matrix of all 1 's and $I$ is the identity matrix. In every subtree representation of $D_{n}$, either the source subtrees have a common vertex and the sink subtrees form an asteroidal collection, or the sink subtrees have a common vertex and the source subtrees form an asteroidal collection.

Proof: Let the vertices of $D_{n}$ be $\{1, \ldots, n\}$; we have $i \rightarrow j$ if and only if $i \neq j$. Consider a subtree representation of $D_{n}$. By Lemma 3, the source subtrees and sink subtrees cannot both be pairwise intersecting; we may assume by symmetry that there is a disjoint pair of source subtrees.

When $i, j, k$ are distinct vertices, we have $i \rightarrow k, j \rightarrow k$, and $k \not \rightarrow k$. Thus Lemma 2 implies that no source subtree can be between two other source subtrees. With betweenness forbidden, $S_{i}$ and $S_{k}$ cannot intersect if $S_{i} \cap S_{j}=\varnothing$. We conclude that if some pair of source subtrees is disjoint, then the source subtrees are pairwise disjoint, and none is between two others. Hence they form an asteroidal collection.

With the source subtrees pairwise disjoint, consider the sink subtrees. For any distinct vertices $i, j, k$, we must have $T_{j}$ containing the path from $S_{i}$ to $S_{k}$ and $T_{i}$ containing the path from $S_{j}$ to $S_{k}$. Hence $T_{i} \cap T_{j} \neq \varnothing$, and the sink subtrees are pairwise intersecting. The Helly property then implies that the sink subtrees have a common vertex.

Together, Lemma 1 and Theorem 2 imply that $l\left(D_{n}\right)=n$.

\section{LEAFAGE AND DISJOINT FERRERS DIMENSION}

We next prove our main lower bound on leafage. We use $N_{D}^{+}(u)$ to denote the successor set and $N_{D}^{-}(u)$ to denote the predecessor set of a vertex $u$ in a digraph $D$.

THEOREM 3. If $D$ is a digraph, then $l(D) \geq f^{*}(D)$.

Proof: Suppose that $l(D)=k$, and let $\left\{\left(S_{v}, T_{v}\right): v \in V(D)\right\}$ be a representation of $D$ in a host tree with $k$ leaves. When $k=2$, the result follows from the characterization of interval digraphs in [15]. For $k \geq 3$, we construct $k$ pairwise disjoint Ferrers digraphs whose union is $\bar{D}$. With the host tree $T$ embedded in the plane, let the leaves be $x_{1}, \ldots, x_{n}$ in clockwise order around the tree. Let $P_{i}$ denote the path in $T$ from $x_{i}$ to $x_{i+1}$, indexed cyclically.

For each leaf $x_{i}$ of the host tree $T$, we construct an associated Ferrers digraph $D(i)$. The edges of $\bar{D}$ consist of those pairs $u v$ such that $S_{u} \cap T_{v}=\varnothing$, meaning that the unique shortest path from $S_{u}$ to $T_{v}$ has length at least 1 . Let $D(i)$ consist of those edges $u v$ in $\bar{D}$ such that the first edge on the path from $S_{u}$ to $T_{v}$ lies on $P_{i}$, with $S_{u}$ between $x_{i}$ and $T_{v}$ (see Fig. 1). 
If $S_{u}$ has no vertex on $P_{i}$, then $u$ has no successors in $D(i)$. If the last vertex of $S_{u^{\prime}}$ on $P_{i}$ is closer to $x_{i+1}$ than the last vertex of $S_{u}$ on $P_{i}$, then $N_{D(i)}^{+}\left(u^{\prime}\right) \subseteq N_{D(i)}^{+}(u)$, by construction. Hence the $D(i)$ 's are Ferrers digraphs.

The paths $P_{i}$ together cover each edge of the host tree exactly once in each direction. Since each edge is covered in each direction, $\bigcup_{i} D(i)=\bar{D}$. Since each edge is covered only once, and when $S_{u} \cap T_{v}=\varnothing$ there is a unique first edge on the path from $S_{u}$ to $T_{v}$, the subgraphs $\{D(i)\}$ are pairwise disjoint.

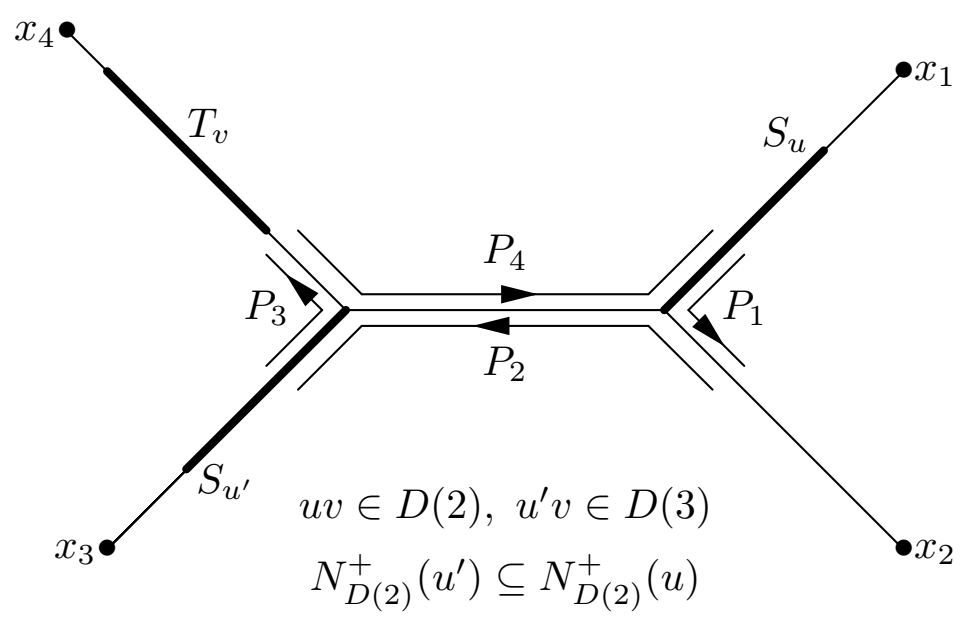

Fig. 1. Ferrers digraphs from subtree representation

This provides another proof that the leafage of the digraph $D_{n}$ is $n$. Since each pair of ones on the diagonal of $A\left(\bar{D}_{n}\right)$ induce a 2 by 2 permutation submatrix, no pair of them can be covered by a single Ferrers digraph contained in $\bar{D}_{n}$.

Although the inequalities $f(D) \leq f^{*}(D) \leq l(D) \leq n(D)$ are best possible, with equality throughout when $D=D_{n}$, the gaps can be arbitrarily large. For an interval digraph, $f(D)=f^{*}(D)=l(D)=2$. By the characterization of interval digraphs in [15], $f^{*}(D)=2$ implies $l(D)=2$. Nevertheless, there exist digraphs $D$ with $f^{*}(D)=3$ and $l(D)=n(D)$.

THEOREM 4. Leafage is not bounded by any function of $f^{*}$ when $f^{*} \geq 3$. In particular, let $E_{n}$ be the $n$-vertex digraph with $A\left(E_{n}\right)=\left(\begin{array}{cc}I & Y \\ Y^{T} & 0\end{array}\right)$, where $I$ denotes the $n-1$ by $n-1$ identity matrix and $\mathrm{Y}$ denotes a column vector of $n-1$ ones. If $n \geq 3$, then $l\left(E_{n}\right)=n$, but $f^{*}\left(E_{n}\right)=f\left(E_{n}\right)=3$.

Proof: Because the last three rows and columns of $A\left(E_{n}\right)$ form a row permutation of $A\left(D_{3}\right)$, we have $f^{*}\left(E_{n}\right) \geq f\left(E_{n}\right) \geq 3$. For equality, partition the zeros of $A\left(\bar{E}_{n}\right)$ into three sets; those in the upper right of the submatrix $I$, those in the lower left of the submatrix $I$, and the 0 in the lower right corner. These sets yield Ferrers digraphs, so $f^{*}\left(E_{n}\right) \leq 3$.

To show that $l\left(E_{n}\right)=n$, we name the vertices by the row and column indices of the matrix and let $\left\{\left(S_{i}, T_{i}\right): 1 \leq i \leq n\right\}$ be a subtree representation of $E_{n}$ in the host tree $T$. By Lemma 1, it suffices to show that the source subtrees or the sink subtrees form an asteroidal collection in $T$. 
We have $S_{n} \cap T_{n}=\varnothing$; let $P$ be the unique path from $S_{n}$ to $T_{n}$ in $T$. For each $k<n$, we have $S_{k} \cap T_{n} \neq \varnothing, S_{n} \cap T_{k} \neq \varnothing$, and $S_{k} \cap T_{k} \neq \varnothing$. Consider also $i<n$. If $P$ contains a vertex $x$ of $S_{i} \cap T_{i}$, then the nonadjacency of $i$ and $k$ implies that $x$ separates $S_{k}$ and $T_{k}$. This contradicts $S_{k} \cap T_{k} \neq \varnothing$, so $S_{i}$ cannot intersect $T_{i}$ in $P$. We conclude that $S_{i} \cap T_{i}$ is contained in the component of $T-E(P)$ containing $T_{n}$ or in the component of $T-E(P)$ containing $S_{n}$. By symmetry, we may assume the latter. Since $n \rightarrow i$, we now have $P \subset T_{i}$. Applying this argument for all vertices other than $n$ yields that all $S_{i} \cap T_{i}$ lie in the same component of $T-E(P)$, since there are no edges except loops among these vertices. Thus $P \subset T_{i}$ and $P \cap S_{i}=\varnothing$ for all $i<n$.

Now consider disjointness and betweenness of the source subtrees. Since $i \rightarrow i, n \rightarrow i$, and $j \not \rightarrow i$, Lemma 2 forbids $S_{j}$ between $S_{i}$ and $S_{n}$ for $i, j<n$. Since $P$ separates $S_{n}$ from the others, this implies that the source subtrees are pairwise disjoint. Furthermore, if $S_{j}$ is between $S_{i}$ and $S_{k}$ for $i, j, k<n$, then the union of the paths from $S_{n}$ to the trees $S_{i}$ and $S_{k}$ must intersect $S_{j}$, which puts $S_{j}$ between $S_{n}$ and one of $\left\{S_{i}, S_{k}\right\}$. Hence the source subtrees are pairwise disjoint, and none is between two others. They form an asteroidal collection, and Lemma 1 applies.

Every $n$ by $n$ (adjacency) matrix with leafage $n$ is a minimal forbidden submatrix for leafage less than $n$. We next present another such family. Given the adjacency matrix $A(D)$ of a digraph $D$, let $H(D)$ be the graph with vertices corresponding to the zeros of $A(D)$ and edges corresponding to the pairs of zeros contains in a 2 by 2 permutation submatrix. Cogis [2] and Doignon-Ducamp-Falmagne [4] proved that $D$ has Ferrers dimension 2 if and only if $H(D)$ is bipartite; here we need only the obvious necessity of the condition.

THEOREM 5. Let $C_{n}$ be the digraph consisting of a directed cycle of length $n$ plus a loop at each vertex. Then $l\left(C_{n}\right)=n$, but $f\left(C_{n}\right)=f^{*}\left(C_{n}\right)=3$.

Proof: Assume that the cycle is $1 \rightarrow 2 \rightarrow \cdots \rightarrow n \rightarrow 1$. Partition the zeros of $A\left(C_{n}\right)$ into three sets: those in the last row, those in the first $n-1$ rows below the diagonal, and the remainder. These sets form Ferrers digraphs, so $f^{*}\left(C_{n}\right) \leq 3$. To prove that $f\left(C_{n}\right)>2$, we observe that the positions

$$
\{(i, i+\lceil n / 2\rceil): 1 \leq i \leq\lfloor n / 2\rfloor\} \cup\{(i, i+1-\lceil n / 2\rceil):\lceil n / 2\rceil \leq i \leq n\}
$$

form an odd cycle in $H\left(C_{n}\right)$.

We use induction on $n$ to prove that $l\left(C_{n}\right)=n$. The claim holds for $n=3$ because $A\left(C_{3}\right)$ is a permutation of $A\left(D_{3}\right)$. For $n>3$, let $\mathbf{T}$ be the host tree for an optimal representation of $C_{n}$. Suppose first that $S_{i-1} \cap S_{i} \neq \varnothing$ for some $i$ (all indexing is circular). The subtree $T_{i}$ must intersect both of these, so by the Helly property $S_{i-1}, T_{i}, S_{i}$ have a common vertex $x$ in $\mathbf{T}$. No other source subtree intersects $T_{i}$, and no other sink subtree intersects $S_{i-1}$ and $S_{i}$; hence no other assigned subtree contains $x$. Every two consecutive subtrees in the list $T_{i+1}, S_{i+1}, T_{i+2}, \ldots, S_{i-2}, T_{i-1}$ intersect; hence their union is connected and contained in one component of $\mathbf{T}-x$. The remaining components of $\mathbf{T}-x$ can be deleted without changing the intersection digraph, so we may assume that $x$ is a leaf.

Let $P$ be the path in $\mathbf{T}$ from $x$ to the nearest branch point. By symmetry, we may assume that $S_{i-1}$ contains as much of $P$ as $S_{i}$. If $S_{i}$ does not contain all of $P$, then $T_{i+1}$ 
intersects $S_{i-1}$, which is forbidden. Hence $P \subseteq S_{i} \cap S_{i-1}$, and no sink subtree other than $T_{i}$ intersects $P$. If another source subtree extends onto $P$, then deleting its edges on $P$ does not change the intersection digraph. We can now delete $T_{i}$ and replace $S_{i-1}, S_{i}$ by a single source subtree with edge set $\left(E\left(S_{i-1}\right) \cup E\left(S_{i}\right)\right)-E(P)$ to obtain a representation of $C_{n-1}$ with $l\left(C_{n}\right)-1$ leaves. By the induction hypothesis, this yields $l\left(C_{n}\right) \geq n$.

Hence we may assume that $S_{i-1} \cap S_{i}=\varnothing$ for all $i$, and by symmetry also $T_{i-1} \cap T_{i}=\varnothing$ for all $i$. In this case, let $P_{i}$ be the portion of $S_{i}$ that is the unique $T_{i}, T_{i+1}$-path, and let $Q_{i}$ be the portion of $T_{i}$ that is the unique $S_{i-1}, S_{i}$-path. Note that $Q_{i} \cap P_{i}$ and $P_{i} \cap Q_{i+1}$ are single vertices. The union of all these paths is thus a closed walk in which no consecutive edges are the same. Such a walk contains a cycle, which is impossible in a host tree. Hence this case does not arise.

We have presented examples with fixed $f^{*}(D)$ and large $l(D)$. Also $f^{*}(D)$ may be arbitrarily large when $f(D)=2$. We construct a two-parameter family of adjacency matrices. The matrix $M_{k, m}$ is a $\mathrm{km}$ by $\mathrm{km}$ matrix consisting of $k$ rows and $k$ columns of $m$ by $m$ blocks. The diagonal blocks are the identity matrix, the blocks below the diagonal consist entirely of 0 's, and the blocks above the diagonal consist entirely of 1's. The zeros can be covered by two Ferrers digraphs, each consisting of all the subdiagonal blocks and half of each diagonal block; hence $f\left(M_{k, m}\right)=2$. We will prove that $f^{*}\left(M_{k, m}\right)=c+1$ when $k=1+\left(\begin{array}{l}c \\ 2\end{array}\right)$ and $m$ is sufficiently large. (In this discussion we use the notation $M_{k, m}$ for both the digraph and its adjacency matrix.)

Let $I_{n}$ denote the $n$-vertex digraph whose adjacency matrix is the identity. A partition of $\bar{I}_{n}$ into $c$ Ferrers digraphs can be viewed as a special $c$-coloring of the 0's in the $n$ by $n$ identity matrix $I_{n}$. We say that colors $A, B$ are a crossed pair if $A, B$ appear together in some row and appear together in some column.

LEMMA 4. If $n \geq 3 c ! / 2$, then every partition of $\bar{I}_{n}$ into $c$ Ferrers digraphs has a crossed pair of colors.

Proof: The proof is by induction on $c$. For $c=2$, a 2-coloring of the 0 's in the 3 by 3 identity matrix cannot have all rows or all columns monochromatic without having a 2 by 2 permutation matrix with 0's in one color. For $c>2$, let $n=3 c ! / 2$ and $r=3(c-1) ! / 2$. Consider a partition of $\bar{I}_{n}$ into $c$ Ferrers digraphs, and suppose that the corresponding coloring has no crossed pair.

Since each row of the identity matrix has $n-10$ 's, the pigeonhole principle implies that each row has at least $\lceil(3(c-1) ! / 2)(c / c)-1 / c\rceil=r$ 's in some color. By symmetry, we may assume there are 0's of color $A$ in the first $r$ columns of row $r+1$ (see Fig. 2 ). Let $D$ be the subdigraph induced by the first $r$ vertices, with $K$ the corresponding submatrix. By the induction hypothesis, every partition of $\bar{D}$ into $c-1$ Ferrers digraphs yields a coloring of the 0 's in $K$ with a crossed pair of colors. Hence we may assume that all $c$ colors (including $A$ ) appear in $K$.

Let $i$ be the index of a row in $K$ in which color $A$ appears. If another color appears in row $i$ of $K$, then it crosses $A$ in the full matrix. Thus we may assume that row $i$ of $K$ has only color $A$. Now, to avoid the forbidden submatrix in color $A$, position $i, r+1$ must have some other color $B$. Now colors $A$ and $B$ appear in a row together, so they cannot 
appear in a column together. This contradicts the observation that every color, including $B$, appears in $K$.

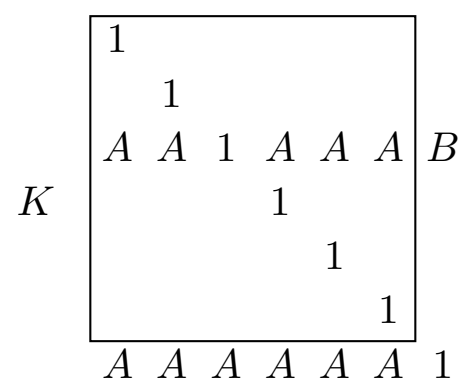

Fig. 2. Coloring 0's in an identity matrix.

The bound $3 c ! / 2$ in Lemma 4 is not best possible. For $c=2,3,4$, the bound is $3,9,36$, but the actual minimum values forcing the desired behavior are $3,4,6$. We are content with the bound arising from the short argument in Lemma 4 because our aim is to show that $f^{*}\left(M_{k, m}\right)$ grows arbitrarily large.

THEOREM 6. If $k \geq 1+\left(\begin{array}{l}c \\ 2\end{array}\right)$ and $m \geq 3 c ! / 2$, then $f^{*}\left(M_{k, m}\right)>c$.

Proof: Suppose $\bar{M}_{k, m}$ has a partition into $c$ pairwise-disjoint Ferrers digraphs. By Lemma 4, in each copy of $I_{m}$ in the block structure of $M_{k, m}$, the corresponding coloring has a crossed pair of colors. Since there are more than $\left(\begin{array}{l}c \\ 2\end{array}\right)$ diagonal blocks, by the pigeonhole principle some pair of colors $A, B$ is crossed twice.

Let $r, s$ be the indices of the diagonal blocks where $A, B$ are crossed, with $r<s$. Let $j$ be the column within diagonal block $r$ where $A, B$ both appear, and let $i$ be the row within diagonal block $s$ where $A, B$ both appear. Position $i, j$ of block $s, r$ is now forced to have both color $A$ and color $B$ to avoid the forbidden substructure for the Ferrers digraphs given by colors $A$ and $B$. This is impossible.

It is worth noting that $f^{*}\left(M_{k, m}\right) \leq c$ for all $m$ when $k \leq\left(\begin{array}{l}c \\ 2\end{array}\right)$. This is illustrated by the block coloring in Fig. 3.

$$
\left(\begin{array}{cccccccccc}
A \backslash B & 1 & 1 & 1 & 1 & 1 & 1 & 1 & 1 & 1 \\
A & A \backslash C & 1 & 1 & 1 & 1 & 1 & 1 & 1 & 1 \\
B & C & B \backslash C & 1 & 1 & 1 & 1 & 1 & 1 & 1 \\
A & A & A & A \backslash D & 1 & 1 & 1 & 1 & 1 & 1 \\
B & B & B & D & B \backslash D & 1 & 1 & 1 & 1 & 1 \\
C & C & C & D & D & C \backslash D & 1 & 1 & 1 & 1 \\
A & A & A & A & A & A & A \backslash E & 1 & 1 & 1 \\
B & B & B & B & B & B & E & B \backslash E & 1 & 1 \\
C & C & C & C & C & C & E & E & C \backslash E & 1 \\
D & D & D & D & D & D & E & E & E & D \backslash E
\end{array}\right)
$$

Fig. 3. A 5-coloring of the 0 's in the blocks of $M_{10, l}$. 
We previously gave examples with $f(D)=3, f^{*}(D)=3$, and $l(D)$ large. We next prove that the family $M_{k, m}$ includes examples with $f(D)=2, f^{*}(D)=3$, and $l(D)$ large.

THEOREM 7. For $m \geq 3, M_{2, m}$ is a $2 m$-vertex digraph with $f\left(M_{2, m}\right)=2, f^{*}\left(M_{2, m}\right)=$ 3 , and $l\left(M_{2, m}\right)=m$.

Proof: With four blocks of order $m, M_{2, m}=\left(\begin{array}{ll}I & 1 \\ 0 & I\end{array}\right)$. The value $f\left(M_{2, m}\right)=2$ was obtained before Lemma 4. The lower bound on $f^{*}$ comes from Theorem 6 (with $c=2$ ), and the upper bound comes from the coloring illustrated in Fig. 3 (with $c=3$ ).

To prove that $l\left(M_{2, m}\right) \leq m$, we construct a representation with $m$ leaves. Let the host tree be the union of $m$ paths $q, s_{i}, t_{i}$ with $q$ as a common endpoint. Let $S_{i}$ and $T_{i+m}$ be the entire $i$ th path, for $1 \leq i \leq m$. Let $S_{i+m}$ be the single vertex $s_{i}$, and let $T_{i}$ be the single vertex $t_{i}$.

It remains to prove that $l\left(M_{2, m}\right) \geq m$. Consider a subtree representation with source subtrees $S_{1}, \ldots, S_{2 m}$ and sink subtrees $T_{1}, \ldots, T_{2 m}$ for the vertices indexed by the rows and columns of $M_{2, m}$ in order. If $T_{m+1}, \ldots, T_{2 m}$ have no common point, then some $T_{i}, T_{j}$ among these are disjoint. Since $T_{i}$ and $T_{j}$ must intersect each of $S_{1}, \ldots, S_{m}$, those subtrees contain the $T_{i}, T_{j}$-path and hence have a common point. Similarly, if $S_{1}, \ldots, S_{m}$ have no common point, then $T_{m+1}, \ldots, T_{2 m}$ must. By symmetry, we may assume that $S_{1}, \ldots, S_{m}$ have a common point $q$.

We now show that $T_{1}, \ldots, T_{m}$ is an asteroidal collection of subtrees. If $T_{i} \cap T_{j} \neq \varnothing$ with $i, j \leq m$, then the entire path from $q$ to the closest vertex of $T_{i} \cap T_{j}$ belongs to at least one of $\left\{S_{i}, S_{j}\right\}$, which contradicts the requirement that each of $\left\{S_{i}, S_{j}\right\}$ intersects exactly one of $\left\{T_{i}, T_{j}\right\}$. If $T_{j}$ is between $T_{i}$ and $T_{k}$, then let $P$ be the path between $T_{i}$ and $T_{k}$, and let $r$ be the vertex of $P$ closest to $q$. Depending on the location of $r$ relative to $T_{j}$ on $P$, the $q, T_{k}$-path in $S_{k}$ or the $q, T_{i}$-path in $S_{i}$ intersects $T_{j}$, contradicting their disjointness from $T_{j}$. Thus $T_{1}, \ldots, T_{m}$ is an asteroidal collection, and Lemma 1 implies that the host has at least $m$ leaves.

\section{CATCH LEAFAGE}

If $D$ has a subtree representation in which every sink subtree is a single vertex, then we say this is a catch representation, and $D$ is a catch-tree digraph. In discussing catch representations, we say "sink point" instead of "sink subtree" to make the usage clear. If $D$ has a catch-tree representation in which the host is a path, then $D$ is a catch-interval digraph. The corresponding classes in which the source sets are single vertices are merely those whose adjacency matrices are the transposes of the digraphs in the classes defined above. Catch-interval digraphs are characterized in [12] under the name "interval catch digraphs" and in [15] under the name "interval-point digraphs".

The catch leafage $l^{*}(D)$ is the minimum number of leaves in a host tree in which $D$ has a catch-tree representation; the catch-interval digraphs are the digraphs with catch-leafage 2 . In the proof of Theorem 1 , we gave every $n$-vertex digraph a catch representation in a star with $n$ leaves, so catch leafage is well-defined. Since every catch-tree representation is a subtree representation, we have $n \geq l^{*}(D) \geq l(D)$. 
We may make several simplifying assumptions about the form of optimal catch-tree representations. In a catch-tree representation, sink subtrees can occupy the same vertex of the host if and only if the corresponding columns of the matrix are identical. We may split such a vertex of the host (without increasing the number of leaves), including the source subtrees to cover both. Thus we may assume that in catch representations each vertex is occupied by at most one sink point. Also, if a vertex of degree at most two in the host tree is not assigned as a sink point, then an edge incident to it can be contracted.

Recall that the predecessor set for $v$ is $N^{-}(v)=\{u: u \rightarrow v\}$; this is the set whose incidence vector is the column of the adjacency matrix corresponding to $v$. Because the source sets occupy single vertices, a catch-tree representation can be described by listing, for each vertex of the host tree, the non-empty collection of source sets containing it. This will be a catch-tree representation if and only if 1) among these sets appear the predecessor sets, and 2) the set of vertices assigned to each source set forms a subtree of the host.

Therefore, our analysis of catch leafage focuses on the columns of the adjacency matrix as incidence vectors for the predecessor sets. We define an associated partial order. Let $P(D)$, the incidence poset of the digraph $D$, be the collection of predecessor sets in $D$, ordered by inclusion. For simplicity, we will use the same notation $V_{j}$ to refer to a predecessor set or the column of the adjacency matrix that is its incidence vector.

The width $w(P)$ of a poset $P$ is the maximum size of its antichains (collections of pairwise incomparable elements). Dilworth's Theorem [3] says that the elements of a finite poset $P$ can be partitioned into $w(P)$ disjoint chains.

THEOREM 8. The inequality $l^{*}(D) \leq w(P(D))$ holds for every digraph $D$ with $w(P(D)) \geq 2$.

Proof: Let $k=w\left(P(D)\right.$, and let $C_{1}, \ldots, C_{k}$ be a partition of $P(D)$ into $k$ disjoint chains. Let the host tree $T$ be a subdivision of a star with $k$ leaves. That is, $T$ consists of a central point of degree $k$ from which $k$ paths emerge. Assign the central vertex the set of all predecessors, and assign to each emerging path the sets on one of the chains $C_{i}$, in decreasing order. The predecessor sets all appear at vertices, and the occurrences of each predecessor form a subtree, so this is a catch-tree representation.

Fulkerson [5] observed that Dilworth's Theorem is equivalent to the König-Egerváry Theorem on matchings in bipartite graphs. Thus bipartite matching or other algorithms can be used to compute $w(P(D))$. Nevertheless, this is only a bound on $l^{*}(D)$, and this bound also can be arbitrarily bad. The digraph $D$ consisting of a directed path plus a loop at each vertex has catch leafage 2 but $w(P(D))=n-1$, so $w(P(D))$ is not bounded by any function of $l^{*}(D)$.

Note that $w(P(D))=1$ when $D$ is a Ferrers digraph. Thus Theorem 8 requires $w(P(D)) \geq 2$, and we see that the break between $w(P(D))$ and $n(D)$ can be large.

We now have the chain of inequalities

$$
f(D) \leq f^{*}(D) \leq l(D) \leq l^{*}(D) \leq w(P(D) \leq n(D) .
$$

One may have equality throughout (achieved by $D_{n}$ ). To prove that there can be arbitrarily bad breaks between any pair, it suffices to produce examples where $l(D)$ is bounded and $l^{*}(D)$ is large. To do this, we prove a sufficient condition for $l^{*}(D)=w(P(D))$. 
THEOREM 9. If $D$ is a digraph such that $P(D)$ has a unique maximal element and $w(P(D)) \geq 2$, then $l^{*}(D)=w(P(D))$.

Proof: Let $V_{0}$ be the unique maximal element, and let $A=V_{1}, \ldots, V_{k}$ denote a maximum antichain in $P(D)$. Let $q_{i}$ denote the vertex of the host assigned to $V_{i}$ in an optimal catch representation. Iteratively delete leaves of the host tree that are not in $\left\{q_{i}\right\}$ until all remaining leaves belong to $\left\{q_{i}\right\}$. If the number of leaves (other than $q_{0}$ ) is less than $k$, then some set $V_{i}$ in $A$ is assigned to a non-leaf $q_{i}$. Every path from $q_{0}$ to another remaining vertex can be extended to reach a remaining leaf. In particular, the path from $q_{0}$ to $q_{i}$ belongs to a path from $q_{0}$ to a leaf assigned $q_{j}$. Since $V_{j} \subseteq V_{0}$ and each predecessor is assigned to the vertices of a tree, this entire path including $q_{i}$ belongs to the source subtrees for $V_{j}$. This yields $V_{j} \subseteq V_{i}$, contradicting the choice of $A$ as an antichain.

THEOREM 10. Catch leafage is not bounded by any function of leafage. If $F_{n}$ denotes the $n$-vertex digraph whose adjacency matrix is $\left(\begin{array}{cc}I & Y \\ Y^{T} & 1\end{array}\right)$, where $I$ denotes the $n-1$ by $n-1$ identity matrix and $\mathrm{Y}$ denotes a column vector of $n-1$ ones, then $f^{*}\left(F_{n}\right)=$ $l\left(F_{n}\right)=2$, but $l^{*}\left(F_{n}\right)=n-1$.

Proof: The upper left and lower right zeros in the portion $I$ of the adjacency matrix yield two disjoint Ferrers digraphs whose union is $\bar{F}_{n}$. As proved in [15], this is equivalent to leafage 2 . On the other hand, the predecessor set of the last vertex contains all the other predecessor sets, so $l^{*}\left(F_{n}\right)=w\left(P\left(F_{n}\right)\right)=n-1$.

The sufficient condition in Theorem 9 does not characterize equality in $l^{*}(D) \leq$ $w(P(D))$. For the digraph $C_{n}$ consisting of a directed cycle plus loops, we have seen that $l\left(C_{n}\right)=n$. Also the columns of $A\left(C_{n}\right)$ form an antichain, so $l\left(C_{n}\right)=l^{*}\left(C_{n}\right)=$ $w\left(P\left(C_{n}\right)\right)=n$.

This example shows also that leafage and catch leafage can drop arbitrarily much when a single vertex is deleted. Deleting one vertex from a cycle with loops leaves a path with loops. The former has leafage and catch leafage $n$; the latter has leafage and catch leafage 2 .

Our proof of $l^{*}(D) \leq w(P(D))$ shows that every digraph has a catch representation in a host tree having only one branch point, and if $P(D)$ has a unique maximum this can be achieved in a host tree with the minimum number of leaves. This is not true of all digraphs. The digraph $D$ with adjacency matrix below contains $C_{4}$ and thus has catch leafage at least 4 . However, every catch representation of $D$ in a host tree with four leaves has two branch points. We thus close by mentioning two further optimization problems for digraphs with catch leafage $k$ : Among catch representations in trees with $k$ leaves, what is the minimum number of branch points, and what is the minimum number of vertices?

$$
\left(\begin{array}{lllll}
1 & 0 & 0 & 1 & 0 \\
1 & 1 & 0 & 0 & 1 \\
0 & 1 & 1 & 0 & 1 \\
0 & 0 & 1 & 1 & 1 \\
1 & 1 & 1 & 1 & 1
\end{array}\right)
$$




\section{References}

[1] L.W. Beineke and C.M. Zamfirescu, Connection digraphs and second order line graphs. Discrete Math. 39(1982), 237-254.

[2] O. Cogis, A characterization of digraphs with Ferrers dimension 2. CNRS Research Report 19(1979).

[3] R.P. Dilworth, A decomposition theorem for partially ordered sets, Ann. Math. 51(1950), 161-165.

[4] J.P. Doignon, A. Ducamp, and J.-C. Falmagne, On realizable biorders and the biorder dimension of a relation. J. Math. Psych. 28(1984), 73-109.

[5] D.R. Fulkerson, Note on Dilworth's decomposition theorem for partially ordered sets. Proc. Amer. Math. Soc. 7(1956), 701-702.

[6] M.C. Golumbic, Algorithmic graph theory and perfect graphs, (Academic Press, 1980).

[7] F. Harary, J.A. Kabell, and F.R. McMorris, Bipartite intersection graphs. Comm. Math. Univ. Carolinae 23(1982), 739-745.

[8] I.-J. Lin, T.A. McKee, and D.B. West, Leafage of chordal graphs, submitted.

[9] I.-J. Lin, M.K. Sen, and D.B. West, Classes of interval digraphs and 0,1-matrices, Congr. Numer. 123-128(1997), to appear.

[10] I.-J. Lin and D.B. West, Interval digraphs that are indifference digraphs. Graph theory, Combinatorics, and Algorithms (Y. Alavi and A. Schwenk, eds.), Proc. 7th Intl. Conf. Graph Th. - Kalamazoo 1992, (Wiley 1995), 751-765.

[11] H. Müller, Recognizing interval digraphs and bi-interval graphs in polynomial time, to appear.

[12] E. Prisner, A characterization of interval catch digraphs, Discrete Math. 73(1989), 285-289.

[13] E. Prisner, Algorithms for interval catch digraphs, 2nd Twente Workshop on Graphs and Combinatorial Optimization (Enschede, 1991), Discrete Appl. Math. 51(1994), 147-157.

[14] J. Riguet, Les relations de Ferrers. Comptes Rendus des Séances hebdomadaires de l'Académie des Sciences (Paris) 232(1951), 1729-1730.

[15] M. Sen, S. Das, A.B. Roy, and D.B. West, Interval digraphs: an analogue of interval graphs. J. Graph Theory 13(1989), 189-202.

[16] M. Sen, S. Das, and D.B. West, Circular-arc digraphs: a characterization. J. Graph Theory 13(1989), 581-592.

[17] M. Sen and B.K. Sanyal, Indifference digraphs: a generalization of indifference graphs. SIAM J. Discr. Math. 7(1994), 157-165.

[18] M. Yannakakis, The complexity of the partial order dimension problem, SIAM J. Algeb. Disc. Meth. 3(1982), 351-328. 